\title{
Semi-regular biorthogonal pairs and generalized Riesz bases
}

\author{
Hiroshi Inoue
}

\begin{abstract}
In this paper we define the notion of semi-regular biorthogonal pairs what is a generalization of regular biorthogonal pairs in Ref. [2] and show that if $\left(\left\{\phi_{n}\right\},\left\{\psi_{n}\right\}\right)$ is a semi-regular biorthogonal pair, then $\left\{\phi_{n}\right\}$ and $\left\{\psi_{n}\right\}$ are generalized Riesz bases. This result improves the results of Ref. [1, 2, 3] in the regular case.
\end{abstract}

\section{Introduction}

Let $\mathcal{H}$ be a Hilbert space with inner product $(\cdot \mid \cdot), \boldsymbol{e}=\left\{e_{n}\right\}$ an ONB in $\mathcal{H}$ and $\left\{\phi_{n}\right\}$ a sequence in $\mathcal{H}$. In Ref. 2], the author has defined an operator $T_{e}$ on $D_{e} \equiv \operatorname{Span}\left\{e_{n}\right\}$ by

$$
T_{\boldsymbol{e}}\left(\sum_{k=0}^{n} \alpha_{k} e_{k}\right)=\sum_{k=0}^{n} \alpha_{k} \phi_{k} .
$$

By using this operator $T_{\boldsymbol{e}}$, the author has investigated the relationship between a regular biorthogonal pair $\left(\left\{\phi_{n}\right\},\left\{\psi_{n}\right\}\right)$ and the notions of Riesz bases and semi-Riesz bases. Here, $\left(\left\{\phi_{n}\right\},\left\{\psi_{n}\right\}\right)$ is a pair of Riesz bases if there exists an ONB $\boldsymbol{e}=\left\{e_{n}\right\}$ in $\mathcal{H}$ such that both $T_{\boldsymbol{e}}$ and $T_{\boldsymbol{e}}^{-1}$ are bounded, and $\left(\left\{\phi_{n}\right\},\left\{\psi_{n}\right\}\right)$ is a pair of semi-Riesz bases if there exists an ONB $\boldsymbol{e}=\left\{e_{n}\right\}$ in $\mathcal{H}$ such that either $T_{e}$ or $T_{e}^{-1}$ are bounded. In this paper we consider the following operators in $\mathcal{H}$ defined by a sequence $\left\{\phi_{n}\right\}$ in $\mathcal{H}$ and an ONB $\boldsymbol{e}=\left\{e_{n}\right\}$ in $\mathcal{H}$ :

$$
\begin{aligned}
T_{\phi, \boldsymbol{e}} & \equiv \sum_{k=0}^{\infty} \phi_{k} \otimes \bar{e}_{k}, \\
T_{\boldsymbol{e}, \phi} & \equiv \sum_{k=0}^{\infty} e_{k} \otimes \bar{\phi}_{k},
\end{aligned}
$$


where the tensor $x \otimes \bar{y}$ of elements $x, y$ of $\mathcal{H}$ is defined by

$$
(x \otimes \bar{y}) \xi=(\xi \mid y) x, \quad \xi \in \mathcal{H} .
$$

This is also denoted by the Dirac notation $|x><y|$. Here we use the notation $x \otimes \bar{y}$.

In Section 2, we investigate the relationship between the operator $T_{\boldsymbol{e}}$ and the operators $T_{\phi, \boldsymbol{e}}$ and $T_{\boldsymbol{e}, \phi}$. The operator $T_{\boldsymbol{e}, \phi}$ is always closed, however $D\left(T_{\phi, \boldsymbol{e}}^{*}\right)$ is not necessarily dense in $\mathcal{H}$, equivalently, $T_{\boldsymbol{e}}$ and $T_{\phi, \boldsymbol{e}}$ are not necessarily closable. Indeed, it is shown that the following statements are equivalent:

(i) $T_{\boldsymbol{e}}$ is closable.

(ii) $T_{\phi, e}$ is closable.

(iii) $D\left(T_{\boldsymbol{e}, \phi}\right)=D(\phi) \equiv\left\{x \in \mathcal{H} ; \sum_{k=0}^{\infty}\left|\left(x \mid \phi_{k}\right)\right|^{2}<\infty\right\}$ is dense in $\mathcal{H}$.

If this holds, then $\bar{T}_{\boldsymbol{e}}=\bar{T}_{\phi, \boldsymbol{e}}=\left(T_{\boldsymbol{e}, \phi}\right)^{*}$.

Furthermore we investigate the relationships between the notion of biorthogonal pairs and the operators $T_{\phi, \boldsymbol{e}}, T_{\boldsymbol{e}, \phi}$. Indeed, if $D(\phi)$ is dense in $\mathcal{H}$, then $\bar{T}_{\phi, \boldsymbol{e}}$ has an inverse and $\bar{T}_{\phi, \boldsymbol{e}}^{-1} \subset T_{\boldsymbol{e}, \psi}=\left(T_{\psi, \boldsymbol{e}}\right)^{*}$. However, $D\left(\bar{T}_{\phi, \boldsymbol{e}}^{-1}\right)$ is not dense in $\mathcal{H}$ in general. And so we may give the conditions under what $D\left(\bar{T}_{\phi, \boldsymbol{e}}^{-1}\right)$ is dense in $\mathcal{H}$. In detail, the following statements are equivalent:

(i) $D_{\phi} \equiv \operatorname{Span}\left\{\phi_{n}\right\}$ is dense in $\mathcal{H}$.

(ii) $T_{\phi, \boldsymbol{e}}$ is closable and $\bar{T}_{\phi, \boldsymbol{e}}$ has a densely defined inverse.

(iii) $T_{\phi, e}^{*}\left(=T_{\boldsymbol{e}, \phi}\right)$ has a densely defined inverse.

If this holds, then $T_{\boldsymbol{e}, \phi}^{-1}=\left(\bar{T}_{\phi, \boldsymbol{e}}^{-1}\right)^{*}$.

In Section 3, we first investigate the relationship between semi-regular biorthogonal pairs and generalized Riesz bases. In Definition 2.1 in Ref [1], the author has defined the notion of generalized Riesz bases under the assumption that $D_{\phi}$ and $D_{\psi}$ are dense in $\mathcal{H}$, and has shown that if $\left(\left\{\phi_{n}\right\},\left\{\psi_{n}\right\}\right)$ is a regular biorthogonal pair, then both $\left\{\phi_{n}\right\}$ and $\left\{\psi_{n}\right\}$ are generalized Riesz bases. In this section, we redefine the notion of generalized Riesz bases, that is, $D_{\phi}$ and $D_{\psi}$ are not necessarily dense in $\mathcal{H}$ and show that if $\left(\left\{\phi_{n}\right\},\left\{\psi_{n}\right\}\right)$ is a semi-regular biorthogonal pair, then both $\left\{\phi_{n}\right\}$ and $\left\{\psi_{n}\right\}$ are generalized Riesz bases. This result improves the results of Ref. [1, 2, 3]. Furthermore, we have the following results:

(i) If $\left(\left\{\phi_{n}\right\},\left\{\psi_{n}\right\}\right)$ is a regular biorthogonal pair, then for any ONB $\boldsymbol{e}=$ $\left\{e_{n}\right\}$ in $\mathcal{H} . \bar{T}_{\phi, \boldsymbol{e}}\left(\right.$ resp. $\left.\bar{T}_{\psi, \boldsymbol{e}}\right)$ is the minimum among constructing operators of the generalized Riesz basis $\left\{\phi_{n}\right\}$ (resp. $\left.\left\{\psi_{n}\right\}\right)$ and $T_{\boldsymbol{e}, \psi}^{-1}\left(\operatorname{resp} . T_{\boldsymbol{e}, \phi}^{-1}\right.$ ) is the 
maximum among constructing operator of $\left\{\phi_{n}\right\}$ (resp. $\left\{\psi_{n}\right\}$ ). Furthermore, any cloesd operator $T$ (resp. $K$ ) satisfying $\bar{T}_{\phi, \boldsymbol{e}} \subset T \subset T_{\boldsymbol{e}, \psi}^{-1}\left(\operatorname{resp} . \bar{T}_{\psi, \boldsymbol{e}} \subset\right.$ $K \subset T_{\boldsymbol{e}, \phi}^{-1}$ ) is a constructing operator for $\left\{\phi_{n}\right\}$ (resp. $\left\{\psi_{n}\right\}$ ).

(ii) If $D(\phi)$ and $D_{\phi}$ are dense in $\mathcal{H}$, then $\bar{T}_{\phi, \boldsymbol{e}}\left(\operatorname{resp} . T_{\boldsymbol{e}, \phi}^{-1}\right)$ is the minimum (resp. the maximum) among constructing operators of $\left\{\phi_{n}\right\}$ (resp. $\left.\left\{\psi_{n}\right\}\right)$.

(iii) If $D(\psi)$ and $D_{\psi}$ are dense in $\mathcal{H}$, then $\bar{T}_{\psi, \boldsymbol{e}}\left(\right.$ resp. $\left.T_{\boldsymbol{e}, \psi}^{-1}\right)$ is the minimum (resp. the maximum) among constructing operators of $\left\{\psi_{n}\right\}$ (resp. $\left.\left\{\phi_{n}\right\}\right)$.

We study the physical operators defined by the operators $T_{\phi, e}, T_{\boldsymbol{e}, \phi}, T_{\psi, \boldsymbol{e}}$ and $T_{\boldsymbol{e}, \psi}$ and an ONB $\boldsymbol{e}=\left\{e_{n}\right\}$. If $D(\phi)$ and $D_{\phi}$ are dense in $\mathcal{H}$, then lowering, raising and number operators $A_{\phi, \boldsymbol{e}}, B_{\phi, \boldsymbol{e}}$ and $N_{\phi, \boldsymbol{e}}$ for $\left\{\phi_{n}\right\}$ are defined, respectively, and raising, lowering and number operators $A_{\boldsymbol{e}, \phi}, B_{\boldsymbol{e}, \phi}$ and $N_{\boldsymbol{e}, \phi}$ for $\left\{\psi_{n}\right\}$ are defined, respectively. Furthermore, if $D(\psi)$ and $D_{\psi}$ are dense in $\mathcal{H}$, then lowering, raising and number operators $A_{\psi, \boldsymbol{e}}, B_{\psi, \boldsymbol{e}}$ and $N_{\psi, \boldsymbol{e}}$ for $\left\{\psi_{n}\right\}$ are defined, respectively, and raising, lowering and number operators $A_{\boldsymbol{e}, \psi}$, $B_{\boldsymbol{e}, \psi}$ and $N_{\boldsymbol{e}, \psi}$ for $\left\{\phi_{n}\right\}$ are defined, respectively. These operators connect with quasi-hermitian quantum mechanics, and its relatives. [10, 7, 5] Many researchers have investigated such operators mathematically. [1, 3, 2, 4]

In Section 4, we shall show a method of constructing a semi-regular biorthogonal pair based on the following commutation rule under some assumptions. Here, the commutation rule is that a pair of operators $a$ and $b$ acting on a Hilbert space $\mathcal{H}$ satisfying

$$
a b-b a=I .
$$

The author has given assumptions to construct the regular biorthogonal pair in Ref. [3]. Indeed, the assumptions in Ref. [3] coincide with the definition of pseudo-bosons as originally given in Ref. [8]. We shall give some assumptions to construct the semi-regular biorthogonal pair that connect with the definition of pseudo-bosons, and show that by using the results in Section 3 and Ref. [3], if $D(\phi)$ and $D_{\phi}$ are dense in $\mathcal{H}$, then we may construct new pseudo-bosonic operators $\left\{A_{\phi, \boldsymbol{e}}, B_{\phi, \boldsymbol{e}}, A_{\boldsymbol{e}, \phi}, B_{\boldsymbol{e}, \phi}\right\}$ and if $D(\psi)$ and $D_{\psi}$ are dense in $\mathcal{H}$, then we may construct a new pseudo-bosonic operators $\left\{A_{\psi, \boldsymbol{e}}, B_{\psi, \boldsymbol{e}}, A_{\boldsymbol{e}, \psi}, B_{\boldsymbol{e}, \psi},\right\}$. Furthermore, we investigate the relationship between pseudo-bosonic operators $\left\{a, b, a^{\dagger}, b^{\dagger}\right\}$ satisfying some assumptions and the operators $\left\{A_{\phi, \boldsymbol{e}}, B_{\phi, \boldsymbol{e}}, A_{\boldsymbol{e}, \phi}, B_{\boldsymbol{e}, \phi}\right\}$ and $\left\{A_{\psi, \boldsymbol{e}}, B_{\psi, \boldsymbol{e}}, A_{\boldsymbol{e}, \psi}, B_{\boldsymbol{e}, \psi},\right\}$. 
This article is organized as follows. In Section 2, we define new operators $T_{\phi, e}$ and $T_{e, \phi}$ and study the property of these operators. Furthermore, we study the relationship between the operator $T_{e}$ and the operators $T_{\phi, \boldsymbol{e}}$ and $T_{\boldsymbol{e}, \phi}$. In Section 3, we investigate the relationship between semiregular biorthogonal pairs and generalized Riesz bases and give the physical operators defined by the operators $T_{\phi, e}, T_{\boldsymbol{e}, \phi}, T_{\psi, \boldsymbol{e}}$ and $T_{\boldsymbol{e}, \psi}$ and an ONB $\boldsymbol{e}=\left\{e_{n}\right\}$. In Section 4, we introduce a method of constructing a semiregular biorthogonal pair based on the pseudo-bosonic operators $\left\{a, b, a^{\dagger}, b^{\dagger}\right\}$ under some assumptions and we investigate the relationship between pseudobosonic operators satisfying some assumptions and the physical operators $\left\{A_{\phi, \boldsymbol{e}}, B_{\phi, \boldsymbol{e}}, A_{\boldsymbol{e}, \phi}, B_{\boldsymbol{e}, \phi}\right\}$ and $\left\{A_{\psi, \boldsymbol{e}}, B_{\psi, \boldsymbol{e}}, A_{\boldsymbol{e}, \psi}, B_{\boldsymbol{e}, \psi},\right\}$. In Section 5, we describe future issue with respect to biorthogonal pairs $\left(\left\{\phi_{n}\right\},\left\{\psi_{n}\right\}\right)$ and generalized Riesz bases.

\section{Some operators defined by biorthogonal se- quences and ONB}

Let $\mathcal{H}$ be a Hilbert space with inner product $(\cdot \mid \cdot)$. We consider the following operators in $\mathcal{H}$ defined by a sequence $\left\{\phi_{n}\right\}$ in a Hilbert space $\mathcal{H}$ and an ONB $\boldsymbol{e}=\left\{e_{n}\right\}$ in $\mathcal{H}$ :

$$
\begin{aligned}
T_{\phi, \boldsymbol{e}} & \equiv \sum_{k=0}^{\infty} \phi_{k} \otimes \bar{e}_{k}, \\
T_{\boldsymbol{e}, \phi} & \equiv \sum_{k=0}^{\infty} e_{k} \otimes \bar{\phi}_{k} .
\end{aligned}
$$

In Ref. 2], the author have defined an operator $T_{\boldsymbol{e}}$ on $D_{\boldsymbol{e}} \equiv \operatorname{Span}\left\{e_{n}\right\}$ by

$$
T_{e}\left(\sum_{k=0}^{n} \alpha_{k} e_{k}\right)=\sum_{k=0}^{n} \alpha_{k} \phi_{k} .
$$

For the operators $T_{\phi, e}, T_{\boldsymbol{e}, \phi}$ and $T_{\boldsymbol{e}}$ we have the following

Lemma 2.1. The following statements hold.

(1) $T_{\phi, e}$ is a densely defined linear operator in $\mathcal{H}$ such that

$$
T_{\phi, e} \supset T_{e} \text { and } T_{\phi, e} e_{n}=\phi_{n}, \quad n=0,1, \cdots .
$$




$$
D\left(T_{\boldsymbol{e}, \phi}\right)=D(\phi) \equiv\left\{x \in \mathcal{H} ; \sum_{k=0}^{\infty}\left|\left(x \mid \phi_{k}\right)\right|^{2}<\infty\right\} \quad \text { and } \quad T_{\boldsymbol{e}}^{*}=T_{\phi, \boldsymbol{e}}^{*}=T_{\boldsymbol{e}, \phi}
$$

Proof. The statements (1) and (2) are easily proved by the definitions of $T_{\phi, \boldsymbol{e}}, T_{\boldsymbol{e}, \phi}$ and $T_{\boldsymbol{e}}$.

By Lemma 2.1, (2), $T_{\boldsymbol{e}, \boldsymbol{\phi}}$ is closed. However $D\left(T_{\phi, \boldsymbol{e}}^{*}\right)$ is not necessarily dense in $\mathcal{H}$, equivalently, $T_{\boldsymbol{e}}$ and $T_{\phi, e}$ are not necessarily closable. Thus we investigate the conditions under what $T_{\phi, e}$ is closable.

Lemma 2.2. The following statements are equivalent:

(i) $T_{\boldsymbol{e}}$ is closable.

(ii) $T_{\phi, \boldsymbol{e}}$ is closable.

(iii) $D(\phi)$ is dense in $\mathcal{H}$.

If this holds, then

$$
\bar{T}_{e}=\bar{T}_{\phi, e}=\left(T_{e, \phi}\right)^{*} .
$$

Proof. This follows from Lemma 2.1, (2).

Next we study the relationships between the notion of biorthogonal pairs and the operators $T_{\phi, \boldsymbol{e}}, T_{\boldsymbol{e}, \phi}$. Then we have the following statements.

Lemma 2.3. Suppose that $\left(\left\{\phi_{n}\right\},\left\{\psi_{n}\right\}\right)$ is a biorthogonal pair such that $D(\phi)$ is dense in $\mathcal{H}$, then $\bar{T}_{\phi, \boldsymbol{e}}$ has an inverse and $\bar{T}_{\phi, \boldsymbol{e}}^{-1} \subset T_{\boldsymbol{e}, \psi}=\left(T_{\psi, \boldsymbol{e}}\right)^{*}$.

Proof. By the definitions of $T_{\phi, \boldsymbol{e}}$ and $T_{\boldsymbol{e}, \psi}$, we have

$$
T_{e, \psi} T_{\phi, e} e_{n}=T_{e, \psi} \phi_{n}=e_{n}, \quad n=0,1, \cdots .
$$

Hence we have

$$
T_{\boldsymbol{e}, \psi} T_{\phi, \boldsymbol{e}}=I \quad \text { on } \quad D_{\boldsymbol{e}}
$$

Thus we have

$$
T_{e, \psi} \bar{T}_{\phi, e}=I
$$


This completes the proof.

In general, $D\left(\bar{T}_{\phi, e}^{-1}\right)$ is not necessarily dense in $\mathcal{H}$. We investigate the conditions under what $D\left(\bar{T}_{\phi, e}^{-1}\right)$ is dense in $\mathcal{H}$.

Lemma 2.4. Suppose that $\left(\left\{\phi_{n}\right\},\left\{\psi_{n}\right\}\right)$ is a biorthogonal pair such that $D(\phi)$ is dense in $\mathcal{H}$. Then the following statements are equivalent:

(i) $D_{\phi} \equiv \operatorname{Span}\left\{\phi_{n}\right\}$ is dense in $\mathcal{H}$.

(ii) $T_{\phi, e}$ is closable and $\bar{T}_{\phi, e}$ has a densely defined inverse.

(iii) $T_{\phi, e}^{*}\left(=T_{e, \phi}\right)$ has a densely defined inverse.

If this holds, then $T_{e, \phi}^{-1}=\left(\bar{T}_{\phi, e}^{-1}\right)^{*}$.

Proof. (i) $\Rightarrow$ (ii) Since $D(\phi)$ is dense in $\mathcal{H}$, by Lemma 2.2 and Lemma 2.3 we have $T_{\phi, \boldsymbol{e}}$ is closable and $\bar{T}_{\phi, \boldsymbol{e}}$ has an inverse. Furthermore, since $D\left(\bar{T}_{\phi, e}^{-1}\right)=\bar{T}_{\phi, e} D\left(\bar{T}_{\phi, e}\right) \supset D_{\phi}$ and $D_{\phi}$ is dense in $\mathcal{H}, \bar{T}_{\phi, e}^{-1}$ is densely defined. (ii) $\Rightarrow$ (iii) By Ref. [2], Lemma 2.2 and Lemma 2.3, we have

$$
\left(\bar{T}_{\phi, \boldsymbol{e}}^{-1}\right)^{*}=\left(\bar{T}_{\phi, \boldsymbol{e}}^{*}\right)^{-1}=\left(T_{\boldsymbol{e}, \phi}\right)^{-1} .
$$

Hence we have

$$
D\left(\left(\bar{T}_{\phi, \boldsymbol{e}}^{*}\right)^{-1}\right)=T_{\boldsymbol{e}, \phi} D\left(T_{\boldsymbol{e}, \phi}\right) \supset T_{\boldsymbol{e}, \phi} D_{\psi}=D_{\boldsymbol{e}} .
$$

Thus we have $\left(T_{\phi, e}^{*}\right)^{-1}$ is densely defined. (iii) $\Rightarrow$ (i) Take an arbitrary $x \in D_{\phi}^{\perp}$. Then,

$$
0=\left(\phi_{n} \mid x\right)=\left(T_{\phi, e} e_{n} \mid x\right)=\left(T_{e} e_{n} \mid x\right), \quad n=0,1, \cdots .
$$

Hence, by Lemma 2.1, (2) we have

$$
x \in D\left(T_{e}^{*}\right)=D\left(T_{\phi, e}^{*}\right) \quad \text { and } \quad T_{e}^{*} x=T_{\phi, e}^{*} x=0 .
$$

By (iii), it follows that

$$
x=\left(T_{\phi, \boldsymbol{e}}^{*}\right)^{-1} T_{\phi, \boldsymbol{e}}^{*} x=0 .
$$

Thus, $D_{\phi}$ is dense in $\mathcal{H}$. This completes the proof. 
Similarly we have the following statements.

Lemma 2.5. Suppose $\left(\left\{\phi_{n}\right\},\left\{\psi_{n}\right\}\right)$ is a biorthogonal pair such that $D(\psi)$ is dense in $\mathcal{H}$. Then the following statements are equivalent:

(i) $D_{\psi} \equiv \operatorname{Span}\left\{\psi_{n}\right\}$ is dense in $\mathcal{H}$.

(ii) $T_{\psi, \boldsymbol{e}}$ is closable and $\bar{T}_{\psi, \boldsymbol{e}}$ has a densely defined inverse.

(iii) $T_{\psi, \boldsymbol{e}}^{*}\left(=T_{\boldsymbol{e}, \psi}\right)$ has a densely defined inverse.

If this holds, then $T_{\boldsymbol{e}, \psi}^{-1}=\left(\bar{T}_{\psi, \boldsymbol{e}}^{-1}\right)^{*}$.

Proof. This is shown similarly to Lemma 2.4 .

\section{Semi-regular biorthogonal pairs and gener- alized Riesz bases}

In Ref. [1], the author has defined the notion of generalized Riesz bases. First we redefine the notion of generalized Riesz bases.

Definition 3.1. If there exists a densely defined closed operator $T$ in $\mathcal{H}$ with a densely defined inverse and there exists an $O N B \boldsymbol{e}=\left\{e_{n}\right\}$ in $\mathcal{H}$ such that

$$
\left\{e_{n}\right\} \subset D(T) \cap D\left(\left(T^{-1}\right)^{*}\right) \quad \text { and } T e_{n}=\phi_{n}, \quad n=0,1, \cdots,
$$

then a sequence $\left\{\phi_{n}\right\}$ in $\mathcal{H}$ is called a generalized Riesz basis with a constructing pair $(\boldsymbol{e}, T)$.

Here, we delete the conditions of Definition 2.1, (ii) and (iii) in Ref. [1, that is, $D_{\phi}$ and $D_{\psi}$ are not necessarily dense in $\mathcal{H}$. Then we have the following

Lemma 3.2. Let $\left\{\phi_{n}\right\}$ be a generalized Riesz basis. Then, we have the following statements.

(1) $T^{*}$ has a densely defined inverse and $\left(T^{*}\right)^{-1}=\left(T^{-1}\right)^{*}$. 
(2) $\psi_{n} \equiv\left(T^{-1}\right)^{*} e_{n}, n=0,1, \cdots$. Then, $\left\{\phi_{n}\right\}$ and $\left\{\psi_{n}\right\}$ are biorthogonal and $\left(T^{-1}\right)^{*}$ is a densely defined closed operator in $\mathcal{H}$ with densely defined inverse $T^{*}$. Hence $\left\{\psi_{n}\right\}$ is a generalized Riesz basis with a constructing pair $\left(\boldsymbol{e},\left(T^{-1}\right)^{*}\right)$.

(3) $D(\phi) \cap D(\psi)$ is dense in $\mathcal{H}$.

Proof. (1) and (2) are easily shown.

(3) We first show that

$$
D\left(T^{*}\right) \subset D(\phi) \text { and } R(T)=D\left(T^{-1}\right) \subset D(\psi) .
$$

Indeed, this follows from

$$
\begin{aligned}
\sum_{k=0}^{\infty}\left|\left(x \mid \phi_{k}\right)\right|^{2} & =\sum_{k=0}^{\infty}\left|\left(T^{*} x \mid e_{k}\right)\right|^{2} \\
& =\left\|T^{*} x\right\|^{2}, \quad x \in D\left(T^{*}\right)
\end{aligned}
$$

and

$$
\begin{aligned}
\sum_{k=0}^{\infty}\left|\left(y \mid \psi_{k}\right)\right|^{2} & =\sum_{k=0}^{\infty}\left|\left(T^{-1} y \mid e_{k}\right)\right|^{2} \\
& =\left\|T^{-1} y\right\|^{2}, \quad y \in D\left(T^{-1}\right) .
\end{aligned}
$$

Since $D\left(T^{*}\right)$ and $R(T)$ are dense in $\mathcal{H}$, it follows that $D(\phi)$ and $D(\psi)$ are dense in $\mathcal{H}$. Next we show that $D(\phi) \cap D(\psi)$ is dense in $\mathcal{H}$. Take an arbitrary $x \in D(T)$. Let $|T|=\int_{0}^{\infty} \lambda d E_{T}(\lambda)$ be the spectral resolution of the absolute $|T| \equiv\left(T^{*} T\right)^{\frac{1}{2}}$ of $T$. Then we have $T E_{T}(n) x \in D\left(T^{*}\right) \cap R(T), n=0,1, \cdots$ and $\lim _{n \rightarrow \infty} T E_{T}(n) x=T x$. Hence $D\left(T^{*}\right) \cap R(T)$ is dense in $R(T)$, and since $R(T)$ is dense in $\mathcal{H}$, it follows from (2.1) that $D(\phi) \cap D(\psi)$ is dense in $\mathcal{H}$. This completes the proof.

In Ref. 2], we have shown that if $\left(\left\{\phi_{n}\right\},\left\{\psi_{n}\right\}\right)$ is a regular biorthogonal pair, then both $\left\{\phi_{n}\right\}$ and $\left\{\psi_{n}\right\}$ are generalized Riesz bases. In order to generalize this result, we define the notion of semi-regular biorthogonal pair as follows:

Definition 3.3. A pair $\left(\left\{\phi_{n}\right\},\left\{\psi_{n}\right\}\right)$ of biorthogonal sequences in $\mathcal{H}$ is said to be semi-regular if either $D(\phi)$ and $D_{\phi}$ are dense in $\mathcal{H}$ or $D(\psi)$ and $D_{\psi}$ 
are dense in $\mathcal{H}$.

We give a concrete example[6] of semi-regular and non regular biorthogonal bases. Let $\left\{e_{n}\right\}$ be an ONB in $\mathcal{H}$ and put $\phi_{n}=e_{n}+e_{0}$ and $\psi_{n}=e_{n}$, $n=1,2, \cdots$. Then it is easily shown that $\left\{\phi_{n}\right\}$ and $\left\{\psi_{n}\right\}$ are biorthogonal bases such that $D_{\phi}$ and $D(\phi)$ are dense in $\mathcal{H}$, but $D_{\psi}$ is not dense in $\mathcal{H}$. We show that if $\left(\left\{\phi_{n}\right\},\left\{\psi_{n}\right\}\right)$ is a semi-regular biorthogonal pair, then both $\left\{\phi_{n}\right\}$ and $\left\{\psi_{n}\right\}$ are generalized Riesz bases. In detail, we have the following

Theorem 3.4. Let $\left\{\phi_{n}\right\}$ and $\left\{\psi_{n}\right\}$ be biorthogonal sequences in $\mathcal{H}$, and let $\boldsymbol{e}=\left\{e_{n}\right\}$ be an arbitrary $O N B$ in $\mathcal{H}$. Then the following statements hold:

(1) Suppose that $\left(\left\{\phi_{n}\right\},\left\{\psi_{n}\right\}\right)$ is a regular biorthogonal pair. Then $\left\{\phi_{n}\right\}$ (resp. $\left.\left\{\psi_{n}\right\}\right)$ is a generalized Riesz basis with constructing pairs $\left(\boldsymbol{e}, \bar{T}_{\phi, \boldsymbol{e}}\right)$ and $\left(\boldsymbol{e}, T_{\boldsymbol{e}, \psi}^{-1}\right)\left(\operatorname{resp} . \quad\left(\boldsymbol{e}, \bar{T}_{\psi, \boldsymbol{e}}\right)\right.$ and $\left.\left(\boldsymbol{e}, T_{\boldsymbol{e}, \phi}^{-1}\right)\right)$, and $\bar{T}_{\phi, \boldsymbol{e}}\left(\operatorname{resp} . \bar{T}_{\psi, \boldsymbol{e}}\right)$ is the minimum among constructing operators of the generalized Riesz basis $\left\{\phi_{n}\right\}$ (resp. $\left.\left\{\psi_{n}\right\}\right)$, and $T_{\boldsymbol{e}, \psi}^{-1}$ (resp. $\left.T_{\boldsymbol{e}, \phi}^{-1}\right)$ is the maximal among constructing operators of $\left\{\phi_{n}\right\}$ (resp. $\left\{\psi_{n}\right\}$ ). Furthermore, any closed operator $T$ (resp. K) satisfying $\bar{T}_{\phi, \boldsymbol{e}} \subset T \subset T_{\boldsymbol{e}, \psi}^{-1}$ (resp. $\left.\bar{T}_{\psi, \boldsymbol{e}} \subset K \subset T_{\boldsymbol{e}, \phi}^{-1}\right)$ is a constructing operator for $\left\{\phi_{n}\right\}$ (resp. $\left.\left\{\psi_{n}\right\}\right)$.

(2) Suppose that $D(\phi)$ and $D_{\phi}$ are dense in $\mathcal{H}$. Then $\left\{\phi_{n}\right\}$ (resp. $\left.\left\{\psi_{n}\right\}\right)$ is a generalized Riesz basis with a constructing pair $\left(\boldsymbol{e}, \bar{T}_{\phi, \boldsymbol{e}}\right)\left(\operatorname{resp} .\left(\boldsymbol{e}, T_{\boldsymbol{e}, \phi}^{-1}\right)\right)$ and the constructing operator $\bar{T}_{\phi, \boldsymbol{e}}$ (resp. $T_{\boldsymbol{e}, \phi}^{-1}$ ) is the minimum (resp. the maximum) among constructing operators of $\left\{\phi_{n}\right\}$ (resp. $\left\{\psi_{n}\right\}$ ).

(3) Suppose that $D(\psi)$ and $D_{\psi}$ are dense in $\mathcal{H}$. Then $\left\{\psi_{n}\right\}$ (resp. $\left.\left\{\phi_{n}\right\}\right)$ is a generalized Riesz basis with a constructing pair $\left(\boldsymbol{e}, \bar{T}_{\psi, \boldsymbol{e}}\right)\left(\operatorname{resp} .\left(\boldsymbol{e}, T_{\boldsymbol{e}, \psi}^{-1}\right)\right.$ ) and the constructing operator $\bar{T}_{\psi, \boldsymbol{e}}$ (resp. $T_{\boldsymbol{e}, \psi}^{-1}$ ) is the minimum (resp. the maximum) among constructing operators of $\left\{\psi_{n}\right\}$ (resp. $\left\{\phi_{n}\right\}$ ).

Proof. Let $\boldsymbol{e}=\left\{e_{n}\right\}$ be any ONB in $\mathcal{H}$.

(1) Since $D(\phi)$ is dense in $\mathcal{H}$, it follows from Lemma 2.3 that $\bar{T}_{\phi, \boldsymbol{e}}$ has an inverse. Since $D_{\phi}$ is also dense in $\mathcal{H}$, it follows from Lemma 2.4 that the inverse $\bar{T}_{\phi, \boldsymbol{e}}^{-1}$ of $\bar{T}_{\phi, \boldsymbol{e}}$ is densely defined. Furthermore, since $\bar{T}_{\phi, \boldsymbol{e}}^{*} \psi_{n}=T_{\boldsymbol{e}, \phi} \psi_{n}=e_{n}$, $n=0,1, \cdots$, we have $\boldsymbol{e} \subset D\left(\left(\bar{T}_{\phi, \boldsymbol{e}}^{*}\right)^{-1}\right)=D\left(T_{\boldsymbol{e}, \phi}^{-1}\right)$. Thus $\left\{\phi_{n}\right\}$ is a generalized Riesz basis with a constructing pair $\left(\boldsymbol{e}, \bar{T}_{\phi, e}\right)$, and $\left\{\psi_{n}\right\}$ is a generalized Riesz basis with a constructing pair $\left(\boldsymbol{e}, T_{\boldsymbol{e}, \phi}^{-1}\right)$. Similarly, $\left\{\psi_{n}\right\}$ is a general- 
ized Riesz basis with a constructing pair $\left(\boldsymbol{e}, \bar{T}_{\psi, e}\right)$, and $\left\{\phi_{n}\right\}$ is a generalized Riesz basis with a constructing pair $\left(\boldsymbol{e}, T_{\boldsymbol{e}, \psi}^{-1}\right)$. Hence $\left\{\phi_{n}\right\}$ (resp. $\left.\left\{\psi_{n}\right\}\right)$ is a generalized Riesz basis with constructing pairs $\left(\boldsymbol{e}, \bar{T}_{\phi, \boldsymbol{e}}\right)$ and $\left(\boldsymbol{e}, T_{\boldsymbol{e}, \psi}^{-1}\right)$ (resp. $\left(\boldsymbol{e}, \bar{T}_{\psi, \boldsymbol{e}}\right)$ and $\left.\left(\boldsymbol{e}, T_{\boldsymbol{e}, \phi}^{-1}\right)\right)$.

Take an arbitrary constructing operator $T$ of the generalized Riesz basis $\left\{\phi_{n}\right\}$. Since $T e_{n}=\phi_{n}$ and $\left(T^{-1}\right)^{*} e_{n}=\psi_{n}, n=0,1, \cdots$, we have $\bar{T}_{\phi, \boldsymbol{e}} \subset T$ and $\bar{T}_{\psi, \boldsymbol{e}} \subset\left(T^{-1}\right)^{*}$, which implies that $T^{-1} \subset T_{\psi, \boldsymbol{e}}^{*}=T_{\boldsymbol{e}, \psi}$. Hence, we have $T \subset T_{e, \psi}^{-1}$. Thus, $\bar{T}_{\phi, e}$ and $T_{e, \psi}^{-1}$ are the minimum and the maximum among constructing operators of $\left\{\phi_{n}\right\}$, respectively. Furthermore, suppose that $T$ is a closed operator in $\mathcal{H}$ such that $\bar{T}_{\phi, e} \subset T \subset T_{e, \psi}^{-1}$. Then, since $D(T) \supset D_{\boldsymbol{e}}$, $T D(T) \supset T_{\phi, \boldsymbol{e}} D_{\boldsymbol{e}}=\left\{\phi_{n}\right\}$ and $D\left(\left(T^{*}\right)^{-1}\right) \supset D\left(T_{\psi, \boldsymbol{e}}\right) \supset D_{\boldsymbol{e}}$, it follows that $T$ is a constructing operator for $\left\{\phi_{n}\right\}$. Similar results for $\left\{\psi_{n}\right\}$ are obtained.

The statements (2) and (3) are shown similarly to (1). This completes the proof.

Remark. Theorem 3.4 means the following:

(1) Suppose $D(\phi)$ and $D_{\phi}\left(\right.$ resp. $D(\psi)$ and $\left.D_{\psi}\right)$ are dense in $\mathcal{H}$. Even if $D_{\psi}\left(\right.$ resp. $\left.D_{\phi}\right)$ is not dense in $\mathcal{H},\left\{\psi_{n}\right\}$ (resp. $\left\{\phi_{n}\right\}$ ) becomes a generalized Riesz basis.

(2) Suppose that $D(\phi)$ and $D_{\phi}$ are dense in $\mathcal{H}$, but $D_{\psi}$ is not dense in $\mathcal{H}$. As shown in Theorem 3.4, $\bar{T}_{\phi, e}$ is the minimum among constructing operators of $\left\{\phi_{n}\right\}$, however the maximal constructing operator of $\left\{\phi_{n}\right\}$ does not necessarily exist because $T_{e, \psi}^{-1}$ is not a constructing operator of $\left\{\phi_{n}\right\}$ different to the case of regular biorthogonal pair. Furthermore, $T_{\boldsymbol{e}, \phi}^{-1}$ is the maximum among constructing operators of $\left\{\psi_{n}\right\}$, however the minimal constructing operator of $\left\{\psi_{n}\right\}$ does not necessarily exist because $\bar{T}_{\psi, e}$ is not a constructing operator of $\left\{\psi_{n}\right\}$. Similar results for the case that $D(\psi)$ and $D_{\psi}$ are dense in $\mathcal{H}$, but $D_{\phi}$ is not dense in $\mathcal{H}$ are obtained.

By Theorem 3.4, Ref. [1] and [3], we can define the physical operators as follows: 
(1) Suppose $D(\phi)$ and $D_{\phi}$ are dense in $\mathcal{H}$. Then, we put

$$
\begin{aligned}
& A_{\phi, \boldsymbol{e}}=\bar{T}_{\phi, \boldsymbol{e}}\left(\sum_{k=0}^{\infty} \sqrt{k+1} e_{k} \otimes \bar{e}_{k+1}\right) \bar{T}_{\phi, \boldsymbol{e}}^{-1}, \\
& B_{\phi, \boldsymbol{e}}=\bar{T}_{\phi, \boldsymbol{e}}\left(\sum_{k=0}^{\infty} \sqrt{k+1} e_{k+1} \otimes \bar{e}_{k}\right) \bar{T}_{\phi, \boldsymbol{e}}^{-1}, \\
& N_{\phi, \boldsymbol{e}}=\bar{T}_{\phi, \boldsymbol{e}}\left(\sum_{k=0}^{\infty} \sqrt{k+1} e_{k+1} \otimes \bar{e}_{k+1}\right) \bar{T}_{\phi, \boldsymbol{e}}^{-1}, \\
& A_{\boldsymbol{e}, \phi}=T_{\boldsymbol{e}, \phi}^{-1}\left(\sum_{k=0}^{\infty} \sqrt{k+1} e_{k+1} \otimes \bar{e}_{k}\right) T_{\boldsymbol{e}, \phi}, \\
& B_{\boldsymbol{e}, \phi}=T_{\boldsymbol{e}, \phi}^{-1}\left(\sum_{k=0}^{\infty} \sqrt{k+1} e_{k} \otimes \bar{e}_{k+1}\right) T_{\boldsymbol{e}, \phi}, \\
& N_{\boldsymbol{e}, \phi}=T_{\boldsymbol{e}, \boldsymbol{\phi}}^{-1}\left(\sum_{k=0}^{\infty} \sqrt{k+1} e_{k+1} \otimes \bar{e}_{k+1}\right) T_{\boldsymbol{e}, \phi} .
\end{aligned}
$$

(2) Suppose $D(\psi)$ and $D_{\psi}$ are dense in $\mathcal{H}$. Then, we put

$$
\begin{aligned}
& A_{\psi, \boldsymbol{e}}=\bar{T}_{\psi, \boldsymbol{e}}\left(\sum_{k=0}^{\infty} \sqrt{k+1} e_{k} \otimes \bar{e}_{k+1}\right) \bar{T}_{\psi, \boldsymbol{e}}^{-1}, \\
& B_{\psi, \boldsymbol{e}}=\bar{T}_{\psi, \boldsymbol{e}}\left(\sum_{k=0}^{\infty} \sqrt{k+1} e_{k+1} \otimes \bar{e}_{k}\right) \bar{T}_{\psi, \boldsymbol{e}}^{-1}, \\
& N_{\psi, \boldsymbol{e}}=\bar{T}_{\psi, \boldsymbol{e}}\left(\sum_{k=0}^{\infty} \sqrt{k+1} e_{k+1} \otimes \bar{e}_{k+1}\right) \bar{T}_{\psi, \boldsymbol{e}}^{-1}, \\
& A_{\boldsymbol{e}, \psi}=T_{\boldsymbol{e}, \psi}^{-1}\left(\sum_{k=0}^{\infty} \sqrt{k+1} e_{k+1} \otimes \bar{e}_{k}\right) T_{\boldsymbol{e}, \psi}, \\
& B_{\boldsymbol{e}, \psi}=T_{\boldsymbol{e}, \psi}^{-1}\left(\sum_{k=0}^{\infty} \sqrt{k+1} e_{k} \otimes \bar{e}_{k+1}\right) T_{\boldsymbol{e}, \psi}, \\
& N_{\boldsymbol{e}, \psi}=T_{\boldsymbol{e}, \psi}^{-1}\left(\sum_{k=0}^{\infty} \sqrt{k+1} e_{k+1} \otimes \bar{e}_{k+1}\right) T_{\boldsymbol{e}, \psi} .
\end{aligned}
$$


Then we have the following

Theorem 3.5. The following statements hold.

(1) Suppose that $D(\phi)$ and $D_{\phi}$ are dense in $\mathcal{H}$. Then we have

$$
\begin{array}{ll}
A_{\phi, e} \phi_{n}=\left\{\begin{array}{cl}
0 & , n=0, \\
\sqrt{n} \phi_{n-1}, & , n=1,2, \cdots,
\end{array}\right. \\
B_{\phi, e} \phi_{n}=\sqrt{n+1} \phi_{n+1} & , n=0,1, \cdots, \\
N_{\phi, e} \phi_{n}=n \phi_{n}, & , n=0,1, \cdots, \\
A_{e, \phi} \psi_{n}=\sqrt{n+1} \psi_{n+1} & , n=0, \\
B_{e, \phi} \psi_{n}=\left\{\begin{array}{cl}
0 & , n=1,2, \cdots, \\
\sqrt{n} \psi_{n-1}, &
\end{array}\right. \\
N_{e, \phi} \psi_{n}=n \psi_{n} .
\end{array}
$$

Hence $A_{\phi, \boldsymbol{e}}, B_{\phi, \boldsymbol{e}}$ and $N_{\phi, \boldsymbol{e}}$ are lowering, raising and number operators for $\left\{\phi_{n}\right\}$, respectively, and $A_{\boldsymbol{e}, \phi}, B_{\boldsymbol{e}, \phi}$ and $N_{\boldsymbol{e}, \phi}$ are raising, lowering and number operators for $\left\{\psi_{n}\right\}$, respectively. 
(2) Suppose that $D(\psi)$ and $D_{\psi}$ are dense in $\mathcal{H}$. Then we have

$$
\begin{array}{ll}
A_{\psi, \boldsymbol{e}} \psi_{n}=\left\{\begin{array}{cl}
0 & , n=0, \\
\sqrt{n} \psi_{n-1}, & , n=1,2, \cdots,
\end{array}\right. \\
B_{\psi, \boldsymbol{e}} \psi_{n}=\sqrt{n+1} \psi_{n+1} & , n=0,1, \cdots, \\
N_{\psi, \boldsymbol{e}} \psi_{n}=n \psi_{n}, & , n=0,1, \cdots, \\
A_{\boldsymbol{e}, \psi} \phi_{n}=\sqrt{n+1} \phi_{n+1} & , n=0, \\
B_{\boldsymbol{e}, \psi} \phi_{n}=\left\{\begin{array}{cl}
0 & , n=1,2, \cdots, \\
\sqrt{n} \phi_{n-1}, &
\end{array}\right. \\
N_{\boldsymbol{e}, \psi} \psi_{n}=n \psi_{n} .
\end{array}
$$

Hence $A_{\psi, e}, B_{\psi, e}$ and $N_{\psi, e}$ are lowering, raising and number operators for $\left\{\psi_{n}\right\}$, respectively, and $A_{\boldsymbol{e}, \psi}, B_{\boldsymbol{e}, \psi}$ and $N_{\boldsymbol{e}, \psi}$ are raising, lowering and number operators for $\left\{\phi_{n}\right\}$, respectively.

Remark.

(i) In case of (1), since

$$
\begin{aligned}
& A_{\boldsymbol{e}, \phi}=\left(T_{\phi, \boldsymbol{e}}^{-1}\right)^{*}\left(\sum_{k=0}^{\infty} \sqrt{k+1} e_{k} \otimes \bar{e}_{k+1}\right)^{*} T_{\phi, \boldsymbol{e}}^{*}, \\
& B_{\boldsymbol{e}, \phi}=\left(T_{\phi, \boldsymbol{e}}^{-1}\right)^{*}\left(\sum_{k=0}^{\infty} \sqrt{k+1} e_{k+1} \otimes \bar{e}_{k}\right)^{*} T_{\phi, \boldsymbol{e}}^{*} \\
& N_{\boldsymbol{e}, \phi}=\left(T_{\phi, \boldsymbol{e}}^{-1}\right)^{*}\left(\sum_{k=0}^{\infty} \sqrt{k+1} e_{k+1} \otimes \bar{e}_{k+1}\right)^{*} T_{\phi, \boldsymbol{e}}^{*}
\end{aligned}
$$

the author has denoted $A_{\boldsymbol{e}}^{\dagger}, B_{\boldsymbol{e}}^{\dagger}$ and $N_{\boldsymbol{e}}^{\dagger}$ in Ref. [3].

(ii) Suppose that $D(\phi)$ and $D_{\phi}$ are dense in $\mathcal{H}$. Then the number operators $N_{\phi, \boldsymbol{e}}$ and $N_{\boldsymbol{e}, \phi}\left(\equiv N_{\phi, \boldsymbol{e}}^{\dagger}\right)$ for $\left\{\phi_{n}\right\}$ and $\left\{\psi_{n}\right\}$, respectively have the relation: $\left(\bar{T}_{\phi, \boldsymbol{e}}^{-1}\right)^{*} \bar{T}_{\phi, \boldsymbol{e}}^{-1} N_{\phi, \boldsymbol{e}}=N_{\phi, \boldsymbol{e}}^{\dagger}\left(\bar{T}_{\phi, \boldsymbol{e}}^{-1}\right)^{*} \bar{T}_{\phi, \boldsymbol{e}}^{-1}$. This is called that $N_{\phi, \boldsymbol{e}}$ is a quasiHermitian operator [11, 12, 9] and positive self-adjoint operator $\left(\bar{T}_{\phi, \boldsymbol{e}}^{-1}\right)^{*} \bar{T}_{\phi, \boldsymbol{e}}^{-1}$ 
is often called a metric operator for the quasi-Hermitian operator $N_{\phi, e}$. Suppose that $D(\psi)$ and $D_{\psi}$ are dense in $\mathcal{H}$. Then the number operator $N_{\boldsymbol{e}, \psi}$ is a quasi-Hermitian operator for the metric operator $\left(\bar{T}_{\psi, \boldsymbol{e}}^{-1}\right)^{*} \bar{T}_{\psi, \boldsymbol{e}}^{-1}$. The results on generalized Riesz bases is related to the problem of finding metric operators for quasi-Hermitian operators.

\section{Semi-regular biorthogonal pairs and Psuedo- bosonic operators}

In this section, we introduce a method of constructing a semi-regular biorthogonal pair based on the following commutation rule under some assumptions. Here, the commutation rule is that a pair of operators $a$ and $b$ acting on a Hilbert space $\mathcal{H}$ with inner product $(\cdot \mid \cdot)$ satisfies

$$
a b-b a=I .
$$

In particular, this collapses to the canonical commutation rule (CCR) if $b=a^{\dagger}$. In Ref. [3] the author has shown assumptions to construct the regular biorthogonal pair. Indeed, the assumptions in Ref. [3] coincide with the definition of pseudo-bosons as originally given in Ref. [8], where in the recent literature many researchers have investigated. [6, 8, 17, 10, 13. In this section, we introduce that some assumptions to construct the semi-regular biorthogonal pair connect with the definition of pseudo-bosons. At first, we construct semi-regular biorthogonal pairs on the above commutation rule. We assume the following statements:

Assumption 1. There exists a non-zero element $\phi_{0}$ of $\mathcal{H}$ such that

(i) $a \phi_{0}=0$,

(ii) $\phi_{0} \in D^{\infty}(b) \equiv \cap_{k=0}^{\infty} D\left(b^{k}\right)$,

(iii) $b^{n} \phi_{0} \in D(a), n=0,1, \cdots$.

Then, we may define a sequence $\left\{\phi_{n}\right\}$ in $\mathcal{H}$ by

$$
\begin{aligned}
\phi_{n} & \equiv \frac{1}{\sqrt{n !}} b^{n} \phi_{0}, \quad n=0,1, \cdots \\
& =\frac{1}{\sqrt{n}} b \phi_{n-1}, \quad n=1,2, \cdots
\end{aligned}
$$


Furthermore, we have the following

Proposition 4.1. The following statements hold.

(1) $b^{n} \phi_{0} \in D\left(a^{m}\right)$ and

$$
a^{m} b^{n} \phi_{0}=\left\{\begin{array}{cl}
{ }_{n} P_{m} b^{n-m} \phi_{0} & , m \leq n, \\
0 & , m>n .
\end{array}\right.
$$

(2) $\phi_{n} \in D\left(N^{m}\right)$ and $N^{m} \phi_{n}=n^{m} \phi_{n}, n, m=0,1, \cdots$. In particular, $N \phi_{n}=n \phi_{n}, n=0,1, \cdots$.

(3)

$$
\begin{array}{ll}
a \phi_{n}= \begin{cases}0 & , n=0, \\
\sqrt{n} \phi_{n-1}, & , n=1,2, \cdots,\end{cases} \\
b \phi_{n}=\sqrt{n+1} \phi_{n+1} & , n=0,1, \cdots .
\end{array}
$$

Proof. These proofs follow from Ref. 3].

Assumption 2. There exists a non-zero element $\psi_{0}$ of $\mathcal{H}$ such that

(i) $b^{\dagger} \psi_{0}=0$,

(ii) $\psi_{0} \in D^{\infty}\left(a^{\dagger}\right) \equiv \cap_{k=0}^{\infty} D\left(\left(a^{\dagger}\right)^{k}\right)$,

(iii) $\left(a^{\dagger}\right)^{n} \psi_{0} \in D\left(b^{\dagger}\right), n=0,1, \cdots$.

Then, we may define a sequence $\left\{\psi_{n}\right\}$ in $\mathcal{H}$ by

$$
\begin{aligned}
\psi_{n} & \equiv \frac{1}{\sqrt{n !}}\left(a^{\dagger}\right)^{n} \psi_{0}, \quad n=0,1, \cdots \\
& =\frac{1}{\sqrt{n}} a^{\dagger} \psi_{n-1}, \quad n=1,2, \cdots .
\end{aligned}
$$

And we put an operator $N^{\dagger} \equiv a^{\dagger} b^{\dagger}$. Furthermore we have the following

Proposition 4.2. The following statements hold.

(1) $\left(a^{\dagger}\right)^{n} \psi_{0} \in D\left(\left(b^{\dagger}\right)^{m}\right)$ and

$$
\left(b^{\dagger}\right)^{m}\left(a^{\dagger}\right)^{n} \psi_{0}=\left\{\begin{array}{cl}
{ }_{n} P_{m}\left(a^{\dagger}\right)^{n-m} \psi_{0} & , m \leq n, \\
0 & , m>n .
\end{array}\right.
$$


(2) $\psi_{n} \in D\left(\left(N^{\dagger}\right)^{m}\right)$ and $\left(N^{\dagger}\right)^{m} \psi_{n}=n^{m} \psi_{n}, n, m=0,1, \cdots$. In particular, $N^{\dagger} \psi_{n}=n \psi_{n}, n=0,1, \cdots$.

(3)

$$
\begin{array}{ll}
a^{\dagger} \psi_{n}=\sqrt{n+1} \psi_{n+1} & , n=0,1, \cdots, \\
b^{\dagger} \psi_{n}=\left\{\begin{array}{cc}
0 & , n=0, \\
\sqrt{n} \psi_{n-1}, & , n=1,2, \cdots .
\end{array}\right.
\end{array}
$$

Proof. These proofs follow from Ref. [3].

The above Assumption 1 and Assumption 2 coincide with the assumptions of Ref. [3]. We weaken the assumption of Ref. [3] to the next assumption in order to construct semi-regular biorthogonal pairs.

\section{Assumption 3.}

Either $D(\phi)$ and $D_{\phi}$ are dense in $\mathcal{H}$ or $D(\psi)$ and $D_{\psi}$ are dense in $\mathcal{H}$.

Then, if a pair of operators $a$ and $b$ acting on $\mathcal{H}$ satisfies Assumption 1$3,\left(\left\{\phi_{n}\right\},\left\{\psi_{n}\right\}\right)$ becomes a semi-regular biorthogonal pair.

By Section 2, Section 3 and Ref. [2, in case of $D(\phi)$ and $D_{\phi}$ are dense in $\mathcal{H}$ (resp. $D(\psi)$ and $D_{\psi}$ are dense in $\left.\mathcal{H}\right), A_{\phi, \boldsymbol{e}}, B_{\phi, \boldsymbol{e}}$ and $N_{\phi, \boldsymbol{e}}$ are lowering, raising and number operators for $\left\{\phi_{n}\right\}$, respectively, and $A_{\boldsymbol{e}, \phi}, B_{\boldsymbol{e}, \phi}$ and $N_{e, \phi}$ are raising, lowering and number operators for $\left\{\psi_{n}\right\}$, respectively. (resp. $A_{\psi, \boldsymbol{e}}, B_{\psi, \boldsymbol{e}}$ and $N_{\psi, \boldsymbol{e}}$ are lowering, raising and number operators for $\left\{\psi_{n}\right\}$, respectively, and $A_{\boldsymbol{e}, \psi}, B_{\boldsymbol{e}, \psi}$ and $N_{\boldsymbol{e}, \psi}$ are raising, lowering and number operators for $\left\{\phi_{n}\right\}$, respectively.). And we have

$$
\begin{aligned}
A_{\phi, \boldsymbol{e}} B_{\phi, \boldsymbol{e}}-B_{\phi, \boldsymbol{e}} A_{\phi, \boldsymbol{e}} \subset I & \text { and } \quad B_{\boldsymbol{e}, \phi} A_{\boldsymbol{e}, \phi}-A_{\boldsymbol{e}, \phi} B_{\boldsymbol{e}, \phi} \subset I . \\
\text { (resp. } \quad A_{\psi, \boldsymbol{e}} B_{\psi, \boldsymbol{e}}-B_{\psi, \boldsymbol{e}} A_{\psi, \boldsymbol{e}} \subset I & \text { and } \left.\quad B_{\boldsymbol{e}, \psi} A_{\boldsymbol{e}, \psi}-A_{\boldsymbol{e}, \psi} B_{\boldsymbol{e}, \psi} \subset I .\right)
\end{aligned}
$$

Furthermore, we have the following statements with respect to the operators $A_{\phi, \boldsymbol{e}}, B_{\phi, \boldsymbol{e}}, A_{\boldsymbol{e}, \phi}$ and $B_{\boldsymbol{e}, \phi}\left(\operatorname{resp} . A_{\psi, \boldsymbol{e}}, B_{\psi, \boldsymbol{e}}, A_{\boldsymbol{e}, \psi}\right.$ and $\left.B_{\boldsymbol{e}, \psi}\right)$. The proofs are easily shown.

Proposition 4.3. If $D(\phi)$ and $D_{\phi}$ are dense in $\mathcal{H}$, then the following statements hold. 
(1)

$$
\begin{aligned}
& \phi_{n}=\frac{1}{\sqrt{n !}} B_{\phi, e}^{n} \phi_{0}, \quad n=0,1, \cdots \\
& \psi_{n}=\frac{1}{\sqrt{n !}} A_{e, \phi}^{n} \psi_{0}, \quad n=0,1, \cdots .
\end{aligned}
$$

$$
\begin{aligned}
& A_{\phi, e} D_{\phi}=D_{\phi}, \quad B_{\phi, e} D_{\phi}=D_{\phi} \\
& \text { and } \\
& A_{\boldsymbol{e}, \phi} D_{\psi}=D_{\psi}, \quad B_{\boldsymbol{e}, \phi} D_{\psi}=D_{\psi} .
\end{aligned}
$$

Proposition 4.4. If $D(\psi)$ and $D_{\psi}$ are dense in $\mathcal{H}$, then the following statements hold.

(1)

$$
\begin{aligned}
& \psi_{n}=\frac{1}{\sqrt{n !}} B_{\psi, \boldsymbol{e}}^{n} \psi_{0}, \quad n=0,1, \cdots, \\
& \phi_{n}=\frac{1}{\sqrt{n !}} A_{e, \psi}^{n} \phi_{0}, \quad n=0,1, \cdots .
\end{aligned}
$$

$$
\begin{array}{ll}
A_{\psi, \boldsymbol{e}} D_{\psi}=D_{\psi}, & B_{\psi, \boldsymbol{e}} D_{\psi}=D_{\psi}, \\
A_{\boldsymbol{e}, \psi} D_{\phi}=D_{\phi}, & \text { and } \quad
\end{array}
$$

Next we investigate the relationship between pseudo-bosonic operators $\left\{a, b, a^{\dagger}, b^{\dagger}\right\}$ satisfying Assumption 1-3 and the operators $A_{\phi, \boldsymbol{e}}, B_{\phi, \boldsymbol{e}}, A_{\boldsymbol{e}, \phi}$ and $B_{\boldsymbol{e}, \phi}\left(A_{\psi, \boldsymbol{e}}\right.$, $B_{\psi, \boldsymbol{e}}, A_{\boldsymbol{e}, \psi}$ and $\left.B_{\boldsymbol{e}, \psi}\right)$.

By Proposition 4.1, Proposition 4.2 and Theorem 3.5 we have the following

Lemma 4.5. The following statements hold.

(1) If $D(\phi)$ and $D_{\phi}$ are dense in $\mathcal{H}$, then $D(a) \cap D(b) \supset D_{\phi}$,

$$
a\left\lceil_ { D _ { \phi } } \subset A _ { \phi , e } \text { and } b \left\lceil_{D_{\phi}} \subset B_{\phi, e} .\right.\right.
$$


(2) If $D(\psi)$ and $D_{\psi}$ are dense in $\mathcal{H}$, then $D\left(a^{\dagger}\right) \cap D\left(b^{\dagger}\right) \supset D_{\psi}$,

$$
a^{\dagger}\left\lceil_ { D _ { \psi } } \subset B _ { \psi , e } \text { and } \quad b ^ { \dagger } \left\lceil_{D_{\psi}} \subset A_{\psi, e} .\right.\right.
$$

Proposition 4.6. The following statements hold.

(1) Suppose that $D(\phi)$ is dense in $\mathcal{H}$ and $D_{\phi}$ is a core for $\bar{a}$ and $\bar{b}$, then $\bar{a} \subset \bar{A}_{\phi, \boldsymbol{e}}$ and $\bar{b} \subset \bar{B}_{\phi, \boldsymbol{e}}$. In particular, if $\bar{T}_{\phi, \boldsymbol{e}}^{-1}$ is bounded, then $\bar{a} \subset A_{\phi, \boldsymbol{e}}=\bar{A}_{\phi, \boldsymbol{e}}$ and $\bar{b} \subset B_{\phi, \boldsymbol{e}}=\bar{B}_{\phi, \boldsymbol{e}}$, and if $\bar{T}_{\phi, \boldsymbol{e}}$ is bounded, then $\bar{a}=\bar{A}_{\phi, \boldsymbol{e}}$ and $\bar{b}=\bar{B}_{\phi, \boldsymbol{e}}$.

(2) Suppose that $D(\psi)$ is dense in $\mathcal{H}$ and $D_{\psi}$ is a core for $\overline{a^{\dagger}}$ and $\overline{b^{\dagger}}$, then $\overline{a^{\dagger}} \subset \bar{B}_{\psi, \boldsymbol{e}}$ and $\overline{b^{\dagger}} \subset \bar{A}_{\psi, \boldsymbol{e}}$. In particular, if $\bar{T}_{\psi, \boldsymbol{e}}^{-1}$ is bounded, then $\overline{a^{\dagger}} \subset B_{\psi, \boldsymbol{e}}=\bar{B}_{\psi, \boldsymbol{e}}$ and $\overline{b^{\dagger}} \subset A_{\psi, \boldsymbol{e}}=\bar{A}_{\psi, \boldsymbol{e}}$, and if $\bar{T}_{\psi, \boldsymbol{e}}$ is bounded, then $\overline{a^{\dagger}}=\bar{B}_{\psi, e}$ and $\overline{b^{\dagger}}=\bar{A}_{\psi, e}$.

Proof. This is shown similarly to Proposition 2.5 in Ref. [3] by using Lemma 4.5.

\section{Discussions}

As shown in Theorem 3.4, if $\left(\left\{\phi_{n}\right\},\left\{\psi_{n}\right\}\right)$ is a semi-regular biorthogonal pair, then $\left\{\phi_{n}\right\}$ and $\left\{\psi_{n}\right\}$ are generalized Riesz bases, and so the physical operators (lowering, raising and number operators) are constructed. In case that $\left(\left\{\phi_{n}\right\},\left\{\psi_{n}\right\}\right)$ is not a semi-regular biorthogonal pair, that is, both $D_{\phi}$ and $D_{\psi}$ are not dense in $\mathcal{H}$, it is meaningful to consider the following question:

Question. Under what conditions is a biorthogonal pair $\left(\left\{\phi_{n}\right\},\left\{\psi_{n}\right\}\right)$ a generalized Riesz basis?

We have estimated that if a biorthogonal pair $\left(\left\{\phi_{n}\right\},\left\{\psi_{n}\right\}\right)$ is a $\mathcal{D}$-quasi basis [6, [5], then $\left\{\phi_{n}\right\}$ and $\left\{\psi_{n}\right\}$ are generalized Riesz bases, where $\mathcal{D}$ is a dense subspace in $\mathcal{H}$ and $\left(\left\{\phi_{n}\right\},\left\{\psi_{n}\right\}\right)$ is a $\mathcal{D}$-quasi basis if

$$
(f, g)=\sum_{k=0}^{\infty}\left(f, \psi_{k}\right)\left(\phi_{k}, g\right)=\sum_{k=0}^{\infty}\left(f, \phi_{k}\right)\left(\psi_{k}, g\right)
$$

for all $f, g \in \mathcal{D}$ 


\section{References}

[1] H. Inoue and M. Takakura, Non-self-adjoint hamiltonians defined by generalized Riesz bases, e-print., arXiv:math-ph/1604.00161

[2] H. Inoue, General theory of regular biorthogonal pairs and its physical applications, e-print., arXiv:math-ph/1604.01967

[3] H. Inoue and M. Takakura, Regular biorthogonal pairs and Psuedobosonic operators, J. Math. Phys., 57(2016), 083503

[4] F. Bagarello, A. Inoue and C. Trapani, Non-self-adjoint hamiltonians defined by Riesz bases, J. Math. Phys., 55(2014), 033501

[5] F. Bagarello, From self to non self-adjoint harmonic oscillators: physical consequences and mathematical pitfalls, Phys. Rev. A., 88(2013), 032120

[6] F. Bagarello, More mathematics for pseudo-bosons, J. Math. Phys., 54(2013), 063512

[7] F. Bagarello, (Regular) pseudo-bosons versus bosons, J. Phys. A., 44(2011), 015205

[8] F. Bagarello, Pseudobosons, Riesz bases, and coherent states, J. Math. Phys., 51(2010), 023531

[9] J. Dieudonné, Quasi-hermitian operators, Proc. Int. Symp. Lin. Spaces, (1961), 115

[10] A. Mostafazadeh, Pseudo-Hermitian representation of Quantum Mechanics, Int. J. Geom. Methods Mod. Phys., 7(2010), 1191-1306

[11] P. Siegl and D. Krejčiřík, On the metric operator for the imaginary cubic oscillator, Phys. Rev. D, 86(2012), 121702(R)

[12] F.G. Scholtz, H.B. Geyer and F.J.W. Hahne, Quasi-Hermitian operators in quantum mechanics and the variational principle, Ann. Phys., 213(2012), 74-101

[13] D.A. Trifonov, Pseudo-boson coherent and Fock states, e-print., arXiv:quant-ph/0902.3744 
Graduate School of Mathematics, Kyushu University, 744 Motooka, Nishiku, Fukuoka 819-0395, Japan

h-inoue@math.kyushu-u.ac.jp, 